Paper ID \#18113

\title{
A Mobile Platform Using Software Defined Radios for Wireless Communica- tion Systems Experimentation
}

\author{
Dr. Otilia Popescu, Old Dominion University
}

Dr. Otilia Popescu received the Engineering Diploma and M.S. degree from the Polytechnic Institute of Bucharest, Romania, and the PhD degree from Rutgers University, all in Electrical and Computer Engineering. Her research interests are in the general areas of communication systems, control theory, signal processing, and engineering education. She is currently an Assistant Professor in the Department of Engineering Technology, Old Dominion University in Norfolk, Virginia. In the past she has worked for the University of Texas at Dallas, University of Texas at San Antonio, Rutgers University, and Politehnica University of Bucharest. She is a senior member of the IEEE, serves as associate editor for IEEE Communication Letters, and has served in the technical program committee for the IEEE ICC, WCNC, RWW, VTC, GLOBECOM, and CAMAD conferences.

\section{Dr. Shiny Abraham, Seattle University}

Shiny Abraham is an Assistant Professor of Electrical and Computer Engineering at Seattle University. She received the B.E. degree in Telecommunication Engineering from Visveswaraiah Technological University (VTU), India in 2007 and Ph.D. from Old Dominion University, Norfolk, VA in 2012. Her research interests span the areas of Wireless Communication, Internet of Things (IoT), Optimization using Game Theory, and Engineering Education Research. She is a member of the IEEE and ASEE, a technical program committee member for IEEE Globecom, ICC, ICCCN and VTC conferences, and a reviewer for several international journals and conferences.

\section{Dr. Samy El-Tawab P.E., James Madison University}

Dr. Samy El-Tawab received his Ph.D. in Computer Science from Old Dominion University, Norfolk, VA, USA in 2012. Dr. El-Tawab is currently an Assistant Professor at James Madison University, Harrisonburg, VA, USA. His main research interests include working on the issues surrounding Intelligent Transportation, (VANET) Vehicular Ad-Hoc Networks, Sensor Networks, Multimedia Communication, Cloud Networks, Voice-over-IP and Computer Security. Dr. El-Tawab introduced a system named "FRIEND": A Cyber-Physical System for Traffic Flow Related Information aggrEgatioN and Dissemination. In 2009, he was awarded a prize for Excellence in Scholarship at The College of William and Mary's 8th Annual Graduate Research Symposium. He has more than 20 publications including journal/conference papers, book chapters, and posters. He also serves as a reviewer in several journals and conferences. http://educ.jmu.edu/ $/$ eltawass/ 


\section{A Mobile Platform Using Software Defined Radios for Wireless Communication Systems Experimentation}

\section{Abstract}

A distinctive feature of wireless communication systems is implied by the fact that there is no physical connection between the transmitter and its corresponding receiver, which enables user mobility. However, experimenting with wireless communication systems is mostly done in the lab, where transmitters and receivers are setup on benches, in stationary settings. This prevents students from experiencing fading and other propagation effects associated with mobile wireless channels. This paper describes a mobile platform for wireless communication experimentation that enables students to run experiments beyond the confines of a traditional lab, in realistic settings that cover indoor and outdoor scenarios with both fixed and mobile propagation characteristics. The platform presented consists of a Universal Software Radio Peripheral (USRP) from National Instruments to implement the transmitter, an affordable RTL-SDR USB dongle to implement the receiver, a laptop computer used to program the SDR boards, and equipment for visualizing radio signal characteristics such as a portable spectrum analyzer or oscilloscope. This choice results in a moderate overall cost for the radio hardware required by the platform, which can be easily programmed using open source software such as GNU Radio as well as software packages like Matlab or LabView. For experimentation in wireless scenarios with low mobility (both indoors and outdoors, corresponding to walking speeds) the transmitter and receiver may be placed on push carts, while for higher mobility they may be placed on university owned golf carts moving at faster speeds on the designated campus routes. Furthermore, mobile transmitters and receivers may also be placed in cars driving on the campus streets and through the university parking lots/garages to enable experiments simulating vehicleto-vehicle (V2V) and vehicle-to-infrastructure (V2I) communications.

\section{Introduction}

Over the past 20 years software defined radio (SDR) platforms have become increasingly considered by researchers and educators alike due to their flexibility, reusable hardware for various set-ups, open source software, short design cycle and accessibility ${ }^{1}$. Hardware prices are within the budget of any engineering department and lately more instructional resources became available ${ }^{2}$, making SDR technology excellent choice for teaching both undergraduate and graduate courses in communications. An example of instructional packages are offered by National Instruments, including hardware platforms, software packages and communication related teaching modules. Integrated curricula with SDR, across areas such as communications, signal processing, computer programming, electromagnetics, and embedded systems, were introduced in six US universities, in each case with a major laboratory component ${ }^{3}$.

Comparisons between course levels, majors, laboratory components, hardware and programming environment used were discussed for the six universities participating and the conclusion of the study was that even though each school has specific ways to implement their courses, the laboratory component and the advantages provided by the SDR hardware for hands-on exploration are very important for student learning. The study was published in 2014 and the authors were concluding by encouraging other schools to use SDR in their courses. Specific implementations of hands-on courses using SDR have been presented by educators and researchers in US and worldwide in different forums ${ }^{4-10}$. The focus of these implementations has 
been augmenting traditional wireless courses with a hands-on component. SDR were used at undergraduate level in senior design projects as well as in term projects ${ }^{4}$, for undergraduate independent study to evaluate the student adaptability to the equipment and easiness to use it to produce results ${ }^{6}$, or to introduce hands-on approach to teach wireless sensor networks at undergraduate level $^{7}$. For sensor networks the Scatterweb's MSB430 sensor platform was preferred ${ }^{7,10}$. From computer engineering perspective, teaching embedded systems for wireless networking was considered, with the use of the microcontrollers MSP430 from Texas Instruments and ARM7-TDMI-S from ARM ${ }^{9}$. SDR platforms were also considered for radar applications ${ }^{11}$.

While the volume of literature on the use of SDR in teaching and research is growing at a fast rate, there is very little coverage on the lab set-up that uses SDR to illustrate the special characteristic of wireless communications systems of no physical connection between transmitter and receiver, which enables mobility and confers wireless networks the advantage over traditional wired networks. Studying the impairments associated with mobile wireless channels by students enrolled in wireless communications courses is challenging, because the labs associated with the course are taught in traditional classroom settings. In such settings, the transmitter and receiver of a wireless link are placed in close proximity to each other, usually on a laboratory bench, which allows students to experiment with modulation schemes commonly used in the context of wireless communication systems, to learn about demodulators and receiver characteristics, and to visualize spectrum occupancy or wireless link performance. While this approach complements the theoretical knowledge that students get during lectures, it also limits their ability to experiment with mobile wireless channels affected by multipath propagation and signal fading. This limitation motivates the use of the mobile platform described in this paper, which is expected to provide students with hands-on experimentation capabilities in realistic settings that cover indoor and outdoor scenarios with both fixed and mobile propagation characteristics. For outdoor wireless scenarios with fixed propagation characteristics, students may study distance-based signal attenuation in wireless communication systems where both the transmitter and the receiver are located on the rooftops of various campus buildings to enable line-of-sight propagation of the radio signals. For mobile propagation studies a small mobile platform would offer special advantages for investigating a variety of scenarios.

In this paper we describe a mobile SDR platform and a set of experiments that are currently under development and testing at Old Dominion University to supplement instruction in wireless communications and networks courses taught in electrical and computer engineering (ECE) and electrical engineering technology (EET) departments. The platform is developed by faculty and graduate students as through an independent study course, and will be formally introduced for classroom demos and research projects starting with the next academic year. Currently the ECE curriculum includes an undergraduate wireless course (400 level) and two graduate wireless course (500 and 600 levels), while the EET curriculum offers a sequence of two undergraduate communication courses (300 and 400 levels), but neither of them offer an actual lab course for communications or wireless communications. In the current structure each of these courses uses class demos based on Matlab, computer assignments and projects for which students work on simulation scenarios that complement the theoretical presentations from the lectures. The development of the mobile platform as described in this paper will enhance the laboratory component of the wireless communication courses even without introducing a laboratory course in the curriculum. Due to its size and portability, the platform can be brought for demos to any classroom where the lectures are scheduled. In a first implementation 2 to 3 lecture classes along 
a semester will be dedicated for experiments involving the platform both indoor and outdoor, with students engaging in the experiments under instructor supervision. The time needed for these classes can be gained by employing the flipped classroom technique for some topics in the course that can be easily covered through pre-recorded videos or other materials. This implementation can be further expanded by introducing assignments or term projects that will require experimenting using the mobile platform. For example, students can team in groups of two or three for a term project, select a particular indoor or outdoor scenario for which they do a beyond the course study through literature, and perform also experiments and data analysis for the same scenario. For average class sizes of up to 30 students the platform can be easily made available upon request for students to perform their experiments. For a curriculum that includes an actual lab course the mobile platform would be a valuable addition to the lab equipment and would be available for a larger range of experiments.

\section{Description of the Wireless Mobile Platform}

The mobile platform for wireless communication systems experimentation described in this paper consists of the following main components:

- A software-defined radio (SDR) board that acts as the radio front-end and includes the antenna and the power supply.

- A laptop computer, which is needed to program the SDR board to act as a specific type of radio, and which is used for information storage and display.

- Specific software packages and associated libraries that are installed on the laptop computer and are required to program the SDR board.

- Other components to be used in conjunction with the mobile platform, which may include optional equipment for signal visualization and performance monitoring, specialized antennas, or mobility elements (moving platform or mobile cart).

\subsection{The Hardware Components}

The hardware elements of the platform are represented by the SDR boards along with the laptop computers needed to program them to act as specific radios. Several types of SDR boards may be used for the mobile platform implementation:

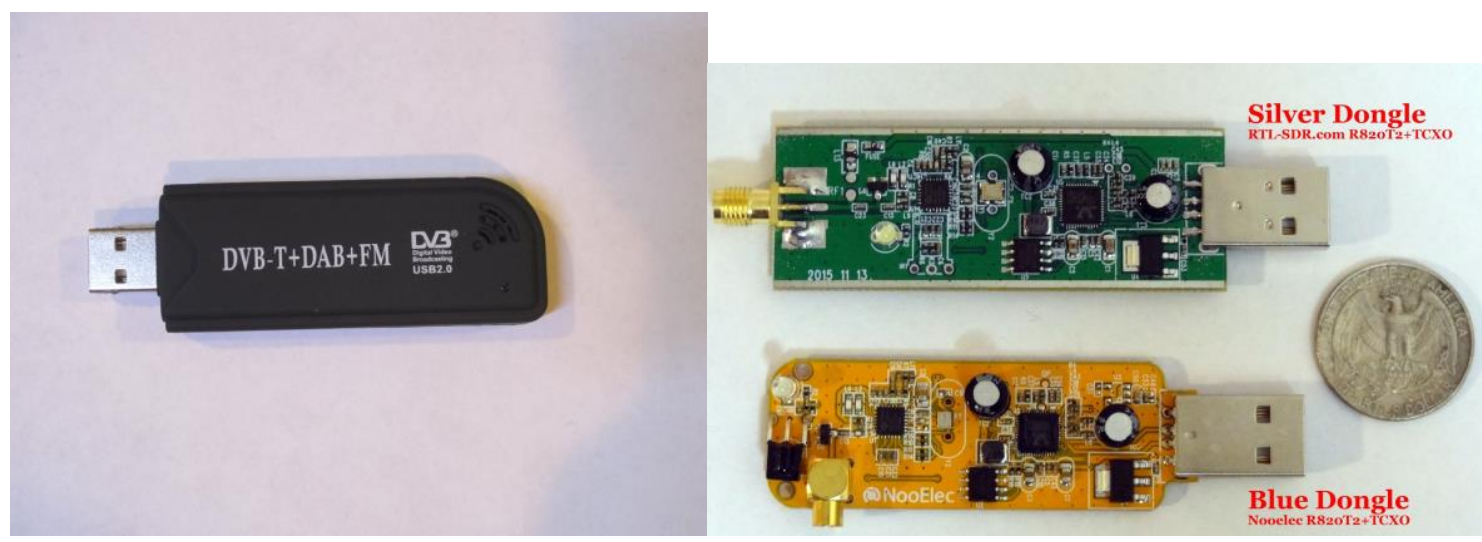

Figure 1. RTL-SDR USB Dongle. [www.radioforeveryone.com, Dec 22, 2016.] 
- The RTL-SDR is a radio receiver based on the Realtek RTL 2832U chip, which operates over a wide band of frequencies, from $24 \mathrm{MHz}$ to $1,766 \mathrm{MHz}$, with a bandwidth of $3.2 \mathrm{MHz}$ and has an 8-bit analog-to-digital converter (ADC) resolution. The RTL-SDR is available in the form of a USB dongle (about the size of a standard USB thumb drive as shown in Figure 1) and provides a cost-effective choice for implementing various types of radio receivers, ranging from FM radio, UHF TV broadcasting, 4G LTE, and GPS systems.

- The Universal Software Radio Platform (USRP) bus series, by Ettus Research (a National Instruments company), is available in two different versions. The USRP1 (shown in Figure 2) is the original motherboard that provides entry-level RF processing capabilities, with an architecture based on an Altera Cyclone FPGA featuring $64 \mathrm{MS} / \mathrm{s}$ dual ADC, $128 \mathrm{MS} / \mathrm{s}$ dual DAC and USB 2.0 connectivity with the host processor. The USRP1 has a modular design, which allows it to operate over a wide range of frequencies spanning DC to $6 \mathrm{GHz}$ by using different types of daughterboards.

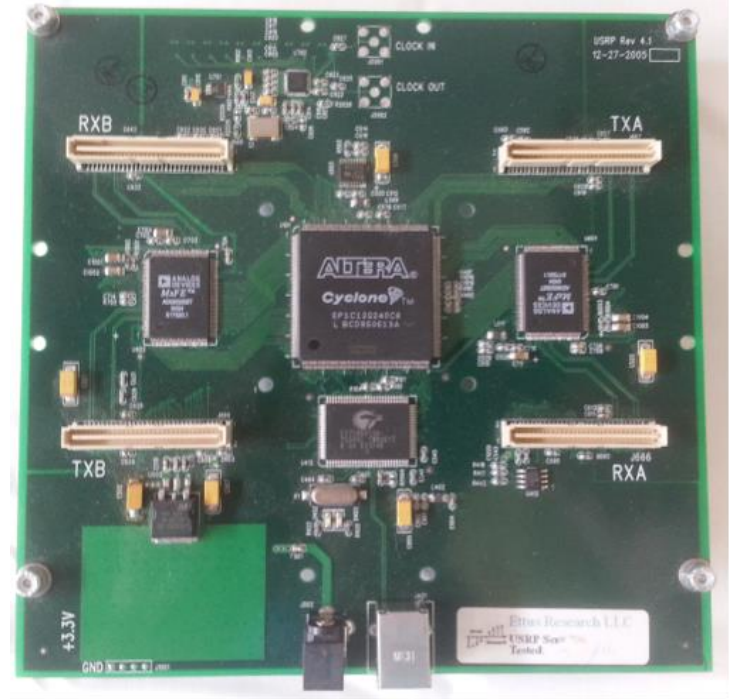

Figure 2. USRP 1 motherboard.

As can be noticed from Figure 2, the USRP1 platform supports two sets of transmit/receive RF daughterboards, TXA/RXA and TXB/RXB. This feature makes the USRP ideal for applications requiring high isolation between transmit and receive chains, or dual-band dual transmit/receive operation. The USRP1 can stream up to $8 \mathrm{MS} / \mathrm{s}$ to and from host applications, and users can implement custom functions in the FPGA fabric.

The different types of daughterboards available for the mobile platform are shown in Figure 3. The TVRX2 daughterboard is ideal for receive-only applications that require access to a number of bands in HF, VHF and UHF frequency ranges, and includes two independent down converter chains that allow for simultaneous reception in two different bands in applications that include dual band receivers for signal collection, whitespace radios, ISM band receivers and broadcast TV reception. 

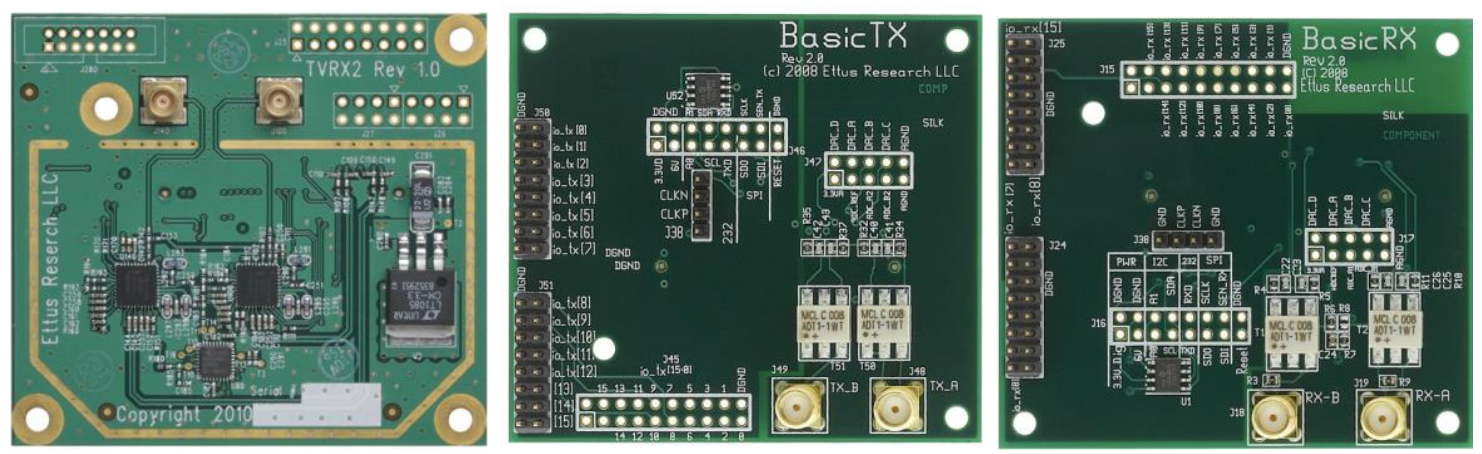

Figure 3. USRP daughterboards: (from left to right) TVRX2, Basic TX, and Basic RX.

The BasicTX and BasicRX daughterboards provide a simple, wideband interface to the digitalto-analog (D/A) and analog-to-digital (A/D) converters of the USRP1 and are ideal for basic applications that require an external RF front with bandwidth ranging from 1 to $250 \mathrm{MHz}$.

The alternative to the USRP1 is the USRP B200 and the B210 shown in Figure 4, which require no additional daughterboards. Figure 5 shows the boards in convenient metallic enclosures that provide protection and flexibility. The USRP B200 provides a fully integrated, single board, Universal Software Radio Peripheral platform with continuous frequency coverage from $70 \mathrm{MHz}$ to $6 \mathrm{GHz}$. Designed for low-cost experimentation, it combines a fully integrated direct conversion transceiver providing up to $56 \mathrm{MHz}$ of real-time bandwidth, an open and reprogrammable Spartan6 FPGA, and fast and convenient bus-powered SuperSpeed USB 3.0 connectivity. The USRP B210 has with 2 transmitters and 2 receivers and allows also MIMO operation and experimentation.
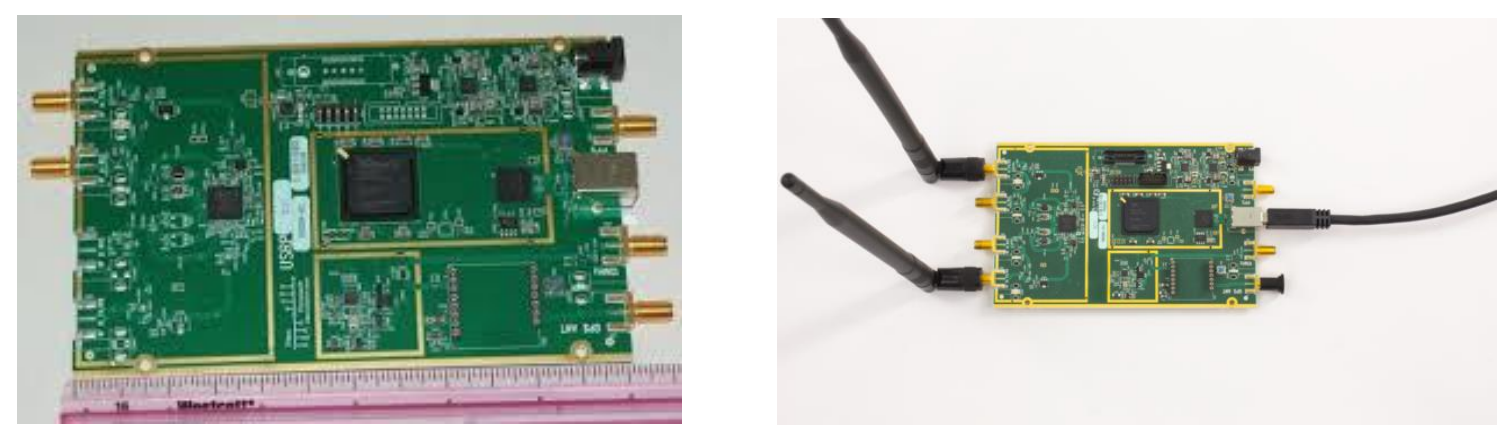

Figure 4. USRP B200 (left) and B210 (right).

Ettus Research also provides a USRP networked series that interfaces with the host processor via a Gigabit Ethernet interface. The most popular of this series is the USRP N200/N210, with an architecture based on the Xilinx® ${ }^{\circledR}$ Spartan® 3A-DSP 3400 FPGA, featuring a100 MS/s dual ADC, and a $400 \mathrm{MS} / \mathrm{s}$ dual DAC. 

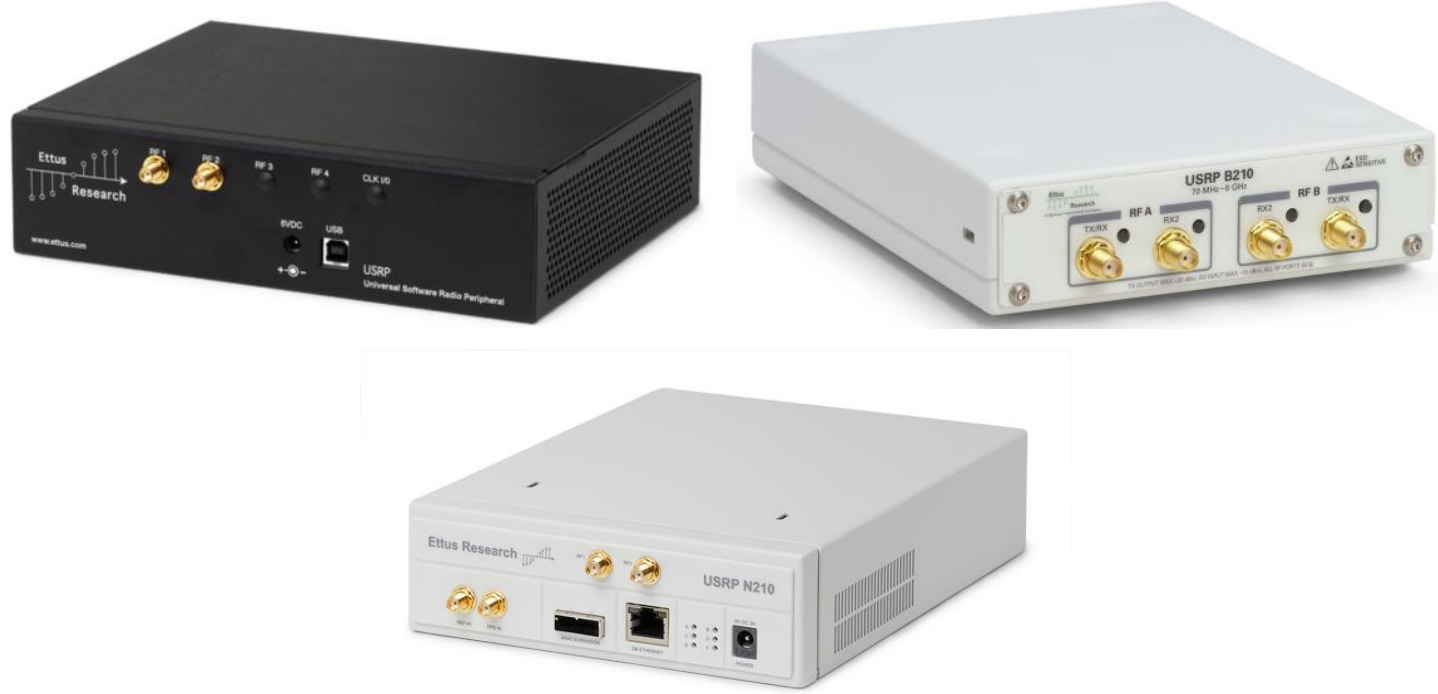

Figure 5. Metallic enclosures for the USRP1 (left), B200/210 (right) and USRP N210 (bottom).

Optional components consisting of equipment for visualizing radio signal characteristics such as a portable spectrum analyzer or oscilloscope may also be included on the platform. In addition, portable antennas with directional characteristics like the ones shown in Figure 6. Portable Yagi antennas (handheld and tripod mounted)., may also be used, to replace the omnidirectional antennas usually used in conjunction with SDR in the lab set-up.
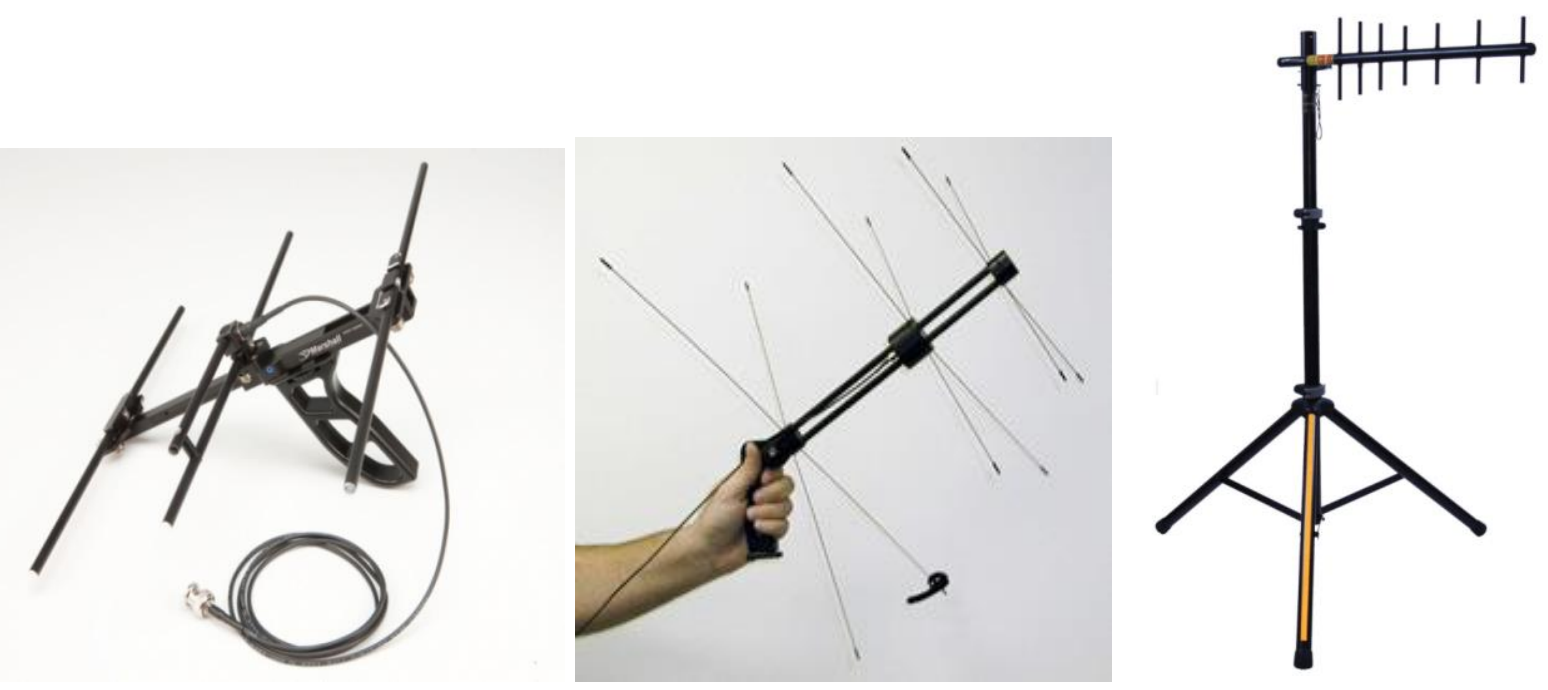

Figure 6. Portable Yagi antennas (handheld and tripod mounted).

\subsection{Host Computer and Enabling Software}

In order to establish the type of radio that the RTL-SDR and the USRP boards will emulate they must be connected to a host computer and programmed using a software package such as Matlab/Simulink, LabView, or the GNU Radio Companion. Since both the RTL-SDR and the USRP boards feature USB connectivity, the obvious choice for a mobile platform is a laptop computer that has at least one USB port available and is capable of running these software packages. We note that for experimentation with the RTL-SDR even an older model Lenovo 
laptop with 32-bit Core 2 processor and running Windows 7 can be used as shown in Figure 7. The same type of laptop may also be used for experimentation with the USRP1, which requires only USB 2.0, while a more recent model having the latest high-speed USB 3.0 will be useful to take full advantage of the USRP B200/210 capabilities.

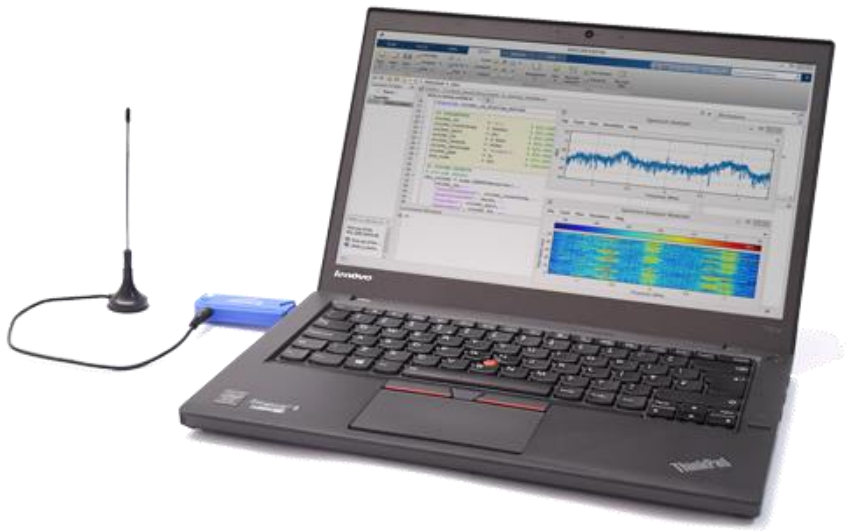

Figure 7 A Lenovo Thinkpad laptop computer with RTL-SDR USB Dongle attached. [http://www.desktopSDR.com/hardware, Dec 22, 2016.]

Before general use laptop computers running the Windows operating system recognize the RTLSDR and the USRP boards, their corresponding drivers must be installed. For RTL-SDR this is done by using the Zadig software, the USRP Hardware Driver (UHD) software provides the required support for programming USRP boards. Once the system drivers are installed, the actual programming of the boards can be accomplished using one of the following software packages:

- Matlab/Simulink is a software package created by MathWorks and used in both academia and industry for machine learning, signal and image processing, computer vision, communications, computational finance, control design, robotics, and much more. Matlab's Communication System Toolbox has support packages for the RTL-SDR and the USRP B200/210 that can be used to design and prototype systems that process real time wireless signals such as FM radio, over-the-air TV, or amateur radio. Unfortunately, Matlab does not provide support for the USRP1 board.

- LabView is a graphical programming environment used by millions of engineers and scientists to develop sophisticated measurement, test, and control systems, which can integrate a variety of hardware devices, including the USRP B200/210 but not the USRP1 board. Furthermore, while National Instruments (NI), which is the supplier of LabView, does not provide support for the RTL-SDR board, some software packages that process signals from the RTL-SDR board are available online, like the SDRLab (http://ha5kfu.sch.bme.hu/sdrlab).

- GNU Radio is an open-source software package that incorporates a graphical tool for creating signal flow graphs and generating flow-graph source code that can be used to program various types of software defined radio platforms, including the RTL-SDR board, the USRP1 and USRP B200/210. We note that GNU Radio is in fact the only software package that can be used to program the USRP1 since neither Matlab nor LabView support the USRP1 board.

The mobile platform developed at Old Dominion University uses the RTL-SDR board to implement narrowband receivers for applications that require up to $3 \mathrm{MHz}$ of bandwidth, while 
the USRP 1 with the TVRX2 daughterboard is used to implement receivers with bandwidth up to $10 \mathrm{MHz}$. For implementing transmitters and duplex links two versatile USRP B200 and B210 are used.

\section{Wireless Communications Experiments}

The main goal of the mobile platform described in this paper is to enable students to run wireless communication experiments beyond the confines of a traditional lab, in realistic settings that cover indoor and outdoor scenarios with both fixed and mobile propagation characteristics.

\subsection{Indoor Experiments}

For indoor studies the experiments should focus on wireless propagation in stationary scenarios and in scenarios with low mobility. For stationary scenarios the positions of the transmitter and the receiver are fixed and students can experiment with power attenuation measurements in order to validate the appropriate wireless channel model. The experiment involves the following main steps, which allow the students to learn about the multipath propagation associated with wireless channels.

- With transmitter and receiver at close distance, the line of sight (LOS) path between transmitter and receiver corresponds to the dominant and the range equation is applicable for propagation modeling.

- Placing the transmitter and receiver further apart in an average size lab room, propagation modeling should include also reflections from the room floor in addition to the LOS component, and a 2-ray multipath propagation model becomes applicable.

- Next, the transmitter and receiver are placed in a long hallway that resembles a dielectric canyon model where a 10-ray multipath propagation model becomes applicable.

- In addition to the multipath propagation effects, by changing the transmitted signal frequency, the students should be able to study the influence of the wireless signal frequency on the different power attenuation models used.

In order to experiment in scenarios with low mobility indoors, the transmitter may be placed in a fixed position at one end of a long hallway while the receiver is placed on a mobile cart that moves towards the transmitter from the other end of the hallway. Alternatively, the mobile receiver cart can be initially located in close proximity to the transmitter and then moved away from it. As the distance between the transmitter and receiver changes the students should also be able to notice variations in the received signal power, which are due to the Doppler Effect that combines with multipath propagation. The transmitter-receiver set-up used for experimentation is illustrated in Figure 8. The flexibility of SDRs can also be leveraged to implement semesteror quarter-long student projects, for example an SDR-based Radio Frequency Identification (RFID) reader and tag. RFID technology has evolved into a ubiquitous wireless application, not just for inventory systems and assets management, but also for healthcare applications (RFID chips being implanted in humans), homeland security and defense applications (security at border crossings). As with any rapidly evolving wireless technology, RFID systems are continually challenged to accommodate new standards and protocols, implement higher security features, and operate in noisy environments. This calls for a transition from conventional hardware radio transmitters and receivers that are limited in their flexibility, cross-functionality, power, and cost-efficiency, to more versatile SDRs. Communication between the reader and tag can be implemented using the USRP N210 along with RF daughterboards RFX900 for the reader and SBX for the tag. 

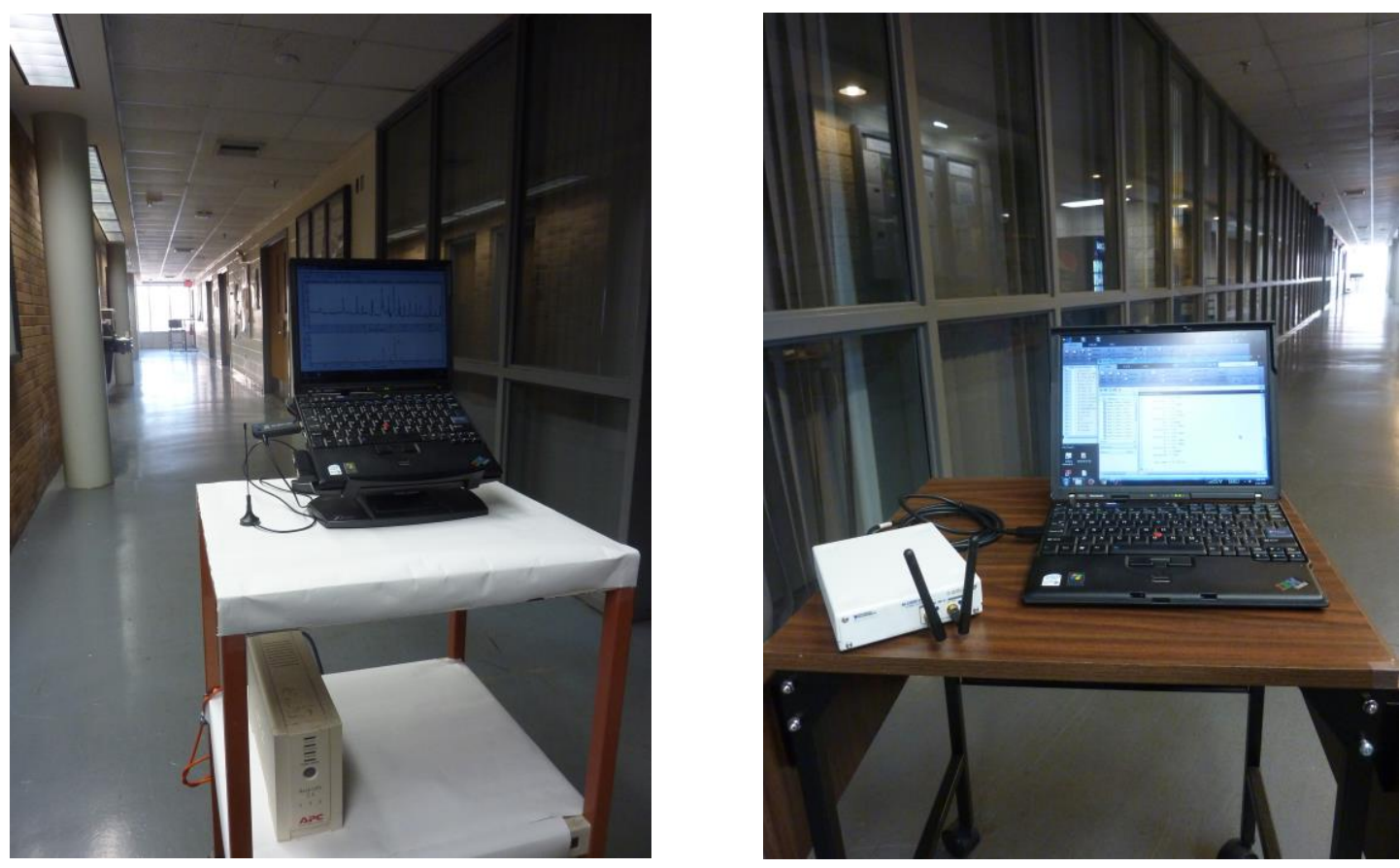

Figure 8. Indoor transmitter-receiver set-up for the mobile platform

\subsection{Outdoor Experiments}

For outdoor studies, the focus is on studying wireless propagation with directional antennas and on experimenting with wireless scenarios with higher mobility. For meaningful experimentation with directional antennas both the transmitter and the receiver are located on the rooftops of tall campus buildings, with a Yagi antenna being used at the transmitter and an omnidirectional antenna at the receiver. This ensures that, when the Yagi antenna is pointed toward the receiver the LOS component of the wireless signal is dominant, while ground reflections due to side lobe transmissions may be neglected. Students should be able to observe how the relative position of the antenna affects propagation, and that the received signal is significantly attenuated when the receiver is located outside the $3-\mathrm{dB}$ beamwidth of the antenna.

For classroom related demos or student research involving experimentation with outdoor mobile wireless scenarios, these can be performed in the university campus, as illustrated in Figure 9, or in a campus parking lot with focus on vehicular communication scenarios. For these experiments the transmitter placed inside a parked vehicle and the receiver placed on a golf cart, or vice versa, such that, if the transmitter is inside the parked vehicle and is stationary one can simulate a vehicle-to-infrastructure (V2I) communication scenario in which the infrastructure transmitter sends information to a moving receiver, while when the transmitter is moving but the receiver is stationary a V2I scenario in which a moving vehicle transmits information to the infrastructure receiver is simulated. 

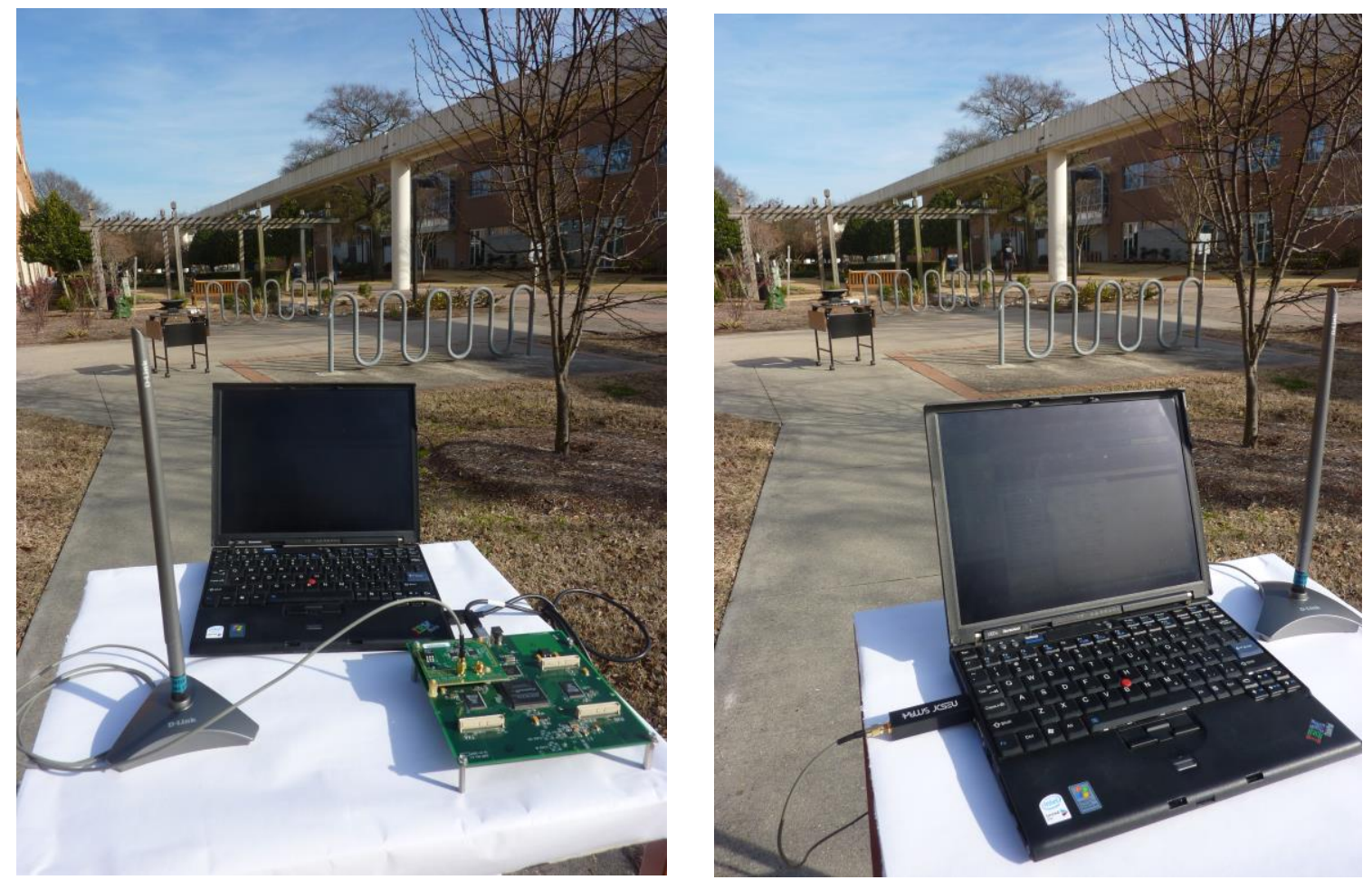

Figure 9. Outdoor transmitter-receiver set-ups for the mobile platform

\section{Conclusions}

In this paper we present a mobile platform for wireless communication experiments that is currently under development and testing at Old Dominion University for use in ECE and EET departments. The platform incorporates SDR boards that are widely available and have become mainstream tools in teaching communication systems. These are used in a novel way to supplement courses in the area of wireless communications and networking by enabling students to study wireless propagation characteristics and perform specific wireless experiments outside traditional labs. By experimenting with the mobile platform students will enhance their hands-on and programming skills, and will become more competitive in the wireless industry job market.

\section{Acknowledgement}

Dr. Otilia Popescu's work was supported in part by the Virginia Space Grant Consortium through the 2015 New Investigator Program.

\section{References}

1. Wyglinski, A. M., Orofino, D. P., Ettus, M. N., \& Rondeau, T. W. (2016). Revolutionizing software defined radio: case studies in hardware, software, and education. IEEE Communications Magazine, 54(1), 68-75.

2. Ge, F., Chen, Q., Wang, Y., Bostian, C. W., Rondeau, T. W., \& Le, B. (2008, March). Cognitive radio: From spectrum sharing to adaptive learning and reconfiguration. In Aerospace Conference, 2008 IEEE (pp. 1-10). IEEE. 
3. Bilén, S. G., Wyglinski, A. M., Anderson, C. R., Cooklev, T., Dietrich, C., FarhangBoroujeny, B., \& Reed, J. H. (2014). Software-defined radio: a new paradigm for integrated curriculum delivery. IEEE Communications Magazine, 52(5), 184-193.

4. Mao, S., Huang, Y., Li, Y., Agrawal, P., \& Tugnait, J. (2013). Introducing software defined radio into undergraduate wireless engineering curriculum through a hands-on approach. In Proc. The 2013 ASEE Annual Conference (pp. 1-10).

5. Bilen, S. (2006, June). Implementing a hands-on course in software-defined radio. In 2006 ASEE Annual Conference Proceedings.

6. Welch, T. B., \& Shearman, S. (2012, March). Teaching software defined radio using the USRP and LabVIEW. In Acoustics, Speech and Signal Processing (ICASSP), 2012 IEEE International Conference on (pp. 2789-2792). IEEE.

7. Förster, A., \& Jazayeri, M. (2010, June). Hands-on approach to teaching wireless sensor networks at the undergraduate level. In Proceedings of the fifteenth annual conference on Innovation and technology in computer science education (pp. 284-288). ACM.

8. Petrova, M., Achtzehn, A., \& Mähönen, P. (2014). System-oriented communications engineering curriculum: teaching design concepts with SDR platforms. IEEE Communications Magazine, 52(5), 202-209.

9. Chenard, J. S., Zilic, Z., \& Prokic, M. (2008). A laboratory setup and teaching methodology for wireless and mobile embedded systems. IEEE Transactions on Education, 51(3), 378-384.

10. Schiller, J., Liers, A., \& Ritter, H. (2005). ScatterWeb: A wireless sensornet platform for research and teaching. Computer Communications, 28(13), 1545-1551.

11. Costanzo, S., Spadafora, F., Di Massa, G., Borgia, A., Costanzo, A., Aloi, G., \& Moreno, O. H. (2013). Potentialities of USRP-based software defined radar systems. Progress in Electromagnetics Research B. 